\title{
Knee kinematics: we need to know more
}

\author{
Jon Karlsson
}

Published online: 24 August 2011

(C) Springer-Verlag 2011

Over and over again, we read about new techniques to reconstruct the anterior cruciate ligament (ACL). In most papers, the authors present interesting and well-thought new surgical techniques. In recent years, novel technical innovations have been discussed over and over again and many of them are sound and improve the patient care, for instance the recent knowledge and new understanding about anatomical ACL reconstruction. As we all know, injuries to the ACL are frequently occurring, especially in young athletes. In fact, an ACL injury is the single most common and serious ligament injury in the body and by far the most costly.

There are still several outstanding questions when it comes to diagnostic evaluation and treatment of ACL injuries. Sometimes we are far too concerned with the surgical techniques and pay too little attention to the patient. Of course, the patient should be in the center all the time.

In this issue of the journal, Decker et al. [1] report on new insights into ACL deficiency and reconstruction through assessment of knee kinematic variability in terms of nonlinear dynamics. In other words, we should be more aware of the possibility of restoring normal knee kinematics and gait. This may happen through rehabilitation or surgery, or preferably both. The authors state that it is critical to better understand neuromuscular function both after an ACL rupture and reconstruction as well and utilize nonlinear measures as an important component of assessment after ACL reconstruction.

The assessment of kinematic variability using nonlinear dynamics can provide important understanding of knee

J. Karlsson $(\bowtie)$

Department of Orthopaedics,

Sahlgrenska University Hospital,

Gothenburg, Sweden

e-mail: Jon.karlsson@telia.com function after ACL injury and after ACL reconstruction. This is of much greater importance than we have previously considered, i.e., not only to understand the surgical anatomy, but also the total function of the entire knee joint, the entire lower limb, and the entire patient.

The authors state that it is important to determine the reliability of nonlinear measures in patients who have sustained an ACL injury, after ACL reconstruction and finally in order to delineate which surgical technique(s) are related to the best outcome in terms of normal (or near normal) nonlinear values.

In other words, we need to pay much more attention to normal mechanics and abnormal neurophysiological issues in patients with ACL injuries. We need to understand the importance of normal knee kinematics during sports activities in ACL-deficient knees and after ACL reconstruction, for instance during gait [2]. This might-in the future-enable us understand some of the mechanisms behind the development of osteoarthritis after an ACL injury and reconstruction. We might also gain more insights into why an ACL reconstruction does not automatically prevent the development of osteoarthritis of the injured knee.

\section{References}

1. Decker LM, Moraiti C, Stergiou N, Georgoulis AD (2011) New insights into anterior cruciate ligament deficiency and reconstruction through the assessment of knee kinematic variability in terms of nonlinear dynamics. Knee Surg Sports Traumatol Arthrosc. doi:10.1007/s00167-011-1484-2

2. Georgoulis AD, Papadonikolakis A, Papageorgiou CD, Mitsou A, Stergiou N (2003) Three-dimensional tibiofemoral kinematics of the anterior cruciate ligament-deficient and reconstructed knee during walking. Am J Sports Med 31:75-79 